a

${ }^{a}$ Dietrich Stauffer Computational Physics Lab, Departamento de Física,Universidade Federal do Piauí, Teresina, Piauí, 64049-550, Brasil 
International Journal of Modern Physics C

(C) World Scientific Publishing Company

\title{
Majority-vote model on Opinion-dependent Network
}

\author{
F. W. S. Lima \\ Dietrich Stauffer Computational Physics Lab, Departamento de Física, Universidade Federal do \\ Piauí, Teresina, Piauí, 64049-550, Brasil ${ }^{\dagger}$ \\ fwslima@gmail.com
}

Received Day Month Year

Revised Day Month Year

\begin{abstract}
We study a nonequilibrium model with up-down symmetry and a noise parameter $q$ known as majority-vote model of M.J. Oliveira 1992 on opinion-dependent network or Stauffer-Hohnisch-Pittnauer networks. By Monte Carlo simulations and finite-size scaling relations the critical exponents $\beta / \nu, \gamma / \nu$, and $1 / \nu$ and points $q_{c}$ and $U^{*}$ are obtained. After extensive simulations, we obtain $\beta / \nu=0.230(3), \gamma / \nu=0.535(2)$, and $1 / \nu=$ $0.475(8)$. The calculated values of the critical noise parameter and Binder cumulant are $q_{c}=0.166(3)$ and $U^{*}=0.288(3)$. Within the error bars, the exponents obey the relation $2 \beta / \nu+\gamma / \nu=1$ and the results presented here demonstrate that the majorityvote model belongs to a different universality class than the equilibrium Ising model on Stauffer-Hohnisch-Pittnauer networks, but to the same class as majority-vote models on some other networks.
\end{abstract}

Keywords: Monte Carlo; Majority vote; Nonequilibrium; Network.

PACS Nos.: 05.10.Ln; 05.70.Fh; 64.60.Fr;

\section{Introduction}

The equilibrium Ising model 12 has become an excellent tool to study models of social application 3. Many of these works are well described in a thorough review 4 , a more recent summary by Stauffer 5 and the following papers in these special issues on sociophysics in this journal. The majority-vote model (MVM) of Oliveira 6 is a nonequilibrium model of social interaction: individuals of a certain population make their decisions based on the opinion of the majority of their neighbors. This model has been studied for several years by various researchers in order to model social and economic systems $\frac{7 / 8|9| 10 \mid 11}{\text { in regular structures } 12|13| 14 \mid 15}$ and various other complex networks $16|17| 18|19| 20|21| 22 \mid 23$.

There are applications to real elections in which similar models of opinion dynamics have been explored in the literature, such as 24 .

\footnotetext{
${ }^{\dagger}$ Piauí, the Universidade Federal do Piauí, Brasil.
} 
In the present work, we study the critical properties of MVM on StaufferHohnisch-Pittnauer (SHP) networks. Hohnisch bonds of SHP networks 25|26|27 are links connecting nodes with different values (spins, opinions, etc.) on them; they are at each time step with a low probability 0.0001 replaced by a link to another randomly selected node. Links connecting agreeing nodes are not replaced. In the present work, we start with each node having links to four randomly selected neighbors. Thus our SHP networks are similar to Small-World (Watts-Strogatz) networks but start from a random network instead of a square lattice and use opinion-dependent (instead of random) rewiring. All links are directed $25 \mid 26$. The critical exponents and noise parameter were obtained using Monte Carlo simulation (MC) and with a finite size scaling analysis. The effective dimension of the SHP network is also determined for MVM. Finally, the critical exponents calculated for SHP networks are compared with the results obtained for undirected and directed Barabási-Albert networks (UBA and DBA) $20 \mid 21$ and Erdös-Rènyi random graphs (UER and DER) 18122 .

\section{Model and simulation}

Our network is SHP type composed of $N$ sites and $k=4$ neighbors. On the MVM model, the system dynamics is as follows. Initially, we assign a spin variable $\sigma$ with values \pm 1 at each node of the network. At each step we try to spin flip a node. The flip is accepted with probability

$$
w_{i}=\frac{1}{2}\left[1-(1-2 q) \sigma_{i} \cdot \mathrm{S}\left(\sum_{j=1}^{k} \sigma_{j}\right)\right],
$$

where $S(x)$ is the sign \pm 1 of $x$ if $x \neq 0, S(x)=0$ if $x=0$. To calculate $w_{i}$ our sum runs over the $k$ nearest neighbors of spin $i$. Eq. (1) means that with probability $(1-q)$ the spin will adopt the same state as the majority of its neighbors.

Here, the control parameter $0 \leq q \leq 1$ plays a role similar to the temperature in equilibrium systems: the smaller $q$, the greater the probability of parallel aligning with the local majority.

To study the critical behavior of the model we define the variable $m \equiv$ $\sum_{i=1}^{N} \sigma_{i} / N$. In particular, we are interested in the magnetization $M$, susceptibility $\chi$ and the reduced fourth-order cumulant $U$

$$
\begin{gathered}
M_{N}(q) \equiv\langle|m|\rangle, \\
\chi_{N}(q) \equiv N\left(\left\langle m^{2}\right\rangle-\langle m\rangle^{2}\right), \\
U_{N}(q) \equiv 1-\frac{\left\langle m^{4}\right\rangle}{3\left\langle m^{2}\right\rangle^{2}},
\end{gathered}
$$

where $\langle\cdots\rangle$ stands for a thermodynamics average. 
The results are averaged over the $N_{\text {run }}$ independent simulations. These quantities are functions of the noise parameter $q$ and obey the finite-size scaling relations

$$
\begin{gathered}
M_{N}(q)=N^{-\beta / \nu} f_{m}(x), \\
\chi_{N}(q)=N^{\gamma / \nu} f_{\chi}(x), \\
\frac{d U_{N}(q)}{d q}=N^{1 / \nu} f_{U}(x),
\end{gathered}
$$

where $\nu, \beta$, and $\gamma$ are the usual critical exponents, $f_{m, \chi, U}(x)$ are the finite size scaling functions with

$$
x=\left(q-q_{c}\right) N^{1 / \nu}
$$

being the scaling variable. Therefore, from the size dependence of $M$ and $\chi$ we obtained the exponents $\beta / \nu$ and $\gamma / \nu$, respectively. The maximum value of susceptibility also scales as $N^{\gamma / \nu}$. Moreover, the value of $q^{*}$ for which $\chi$ has a maximum is expected to scale with the system size $N$ as

$$
q^{*}=q_{c}+b N^{-1 / \nu} \text { with } b \approx 1 .
$$

Therefore, the relations (3c) and (4) may be used to get the exponent $1 / \nu$. We also have applied the calculated exponents to the hyperscaling hypothesis

$$
2 \beta / \nu+\gamma / \nu=D_{\text {eff }}
$$

in order to get the effective dimensionality, $D_{e f f}$, for connectivity $k$.

We performed Monte Carlo simulation on the SHP networks with various systems sizes $N(250,500,1000,2000,4000,8000$ and 16000 sites $)$. It takes $2 \times 10^{5}$ Monte Carlo steps (MCS) to make the system reach the steady state, and then the time averages are estimated over the next $2 \times 10^{5}$ MCS. One MCS is accomplished after all the $N$ spins are investigated whether they flip or not.

The results are averaged over $N_{\text {run }}\left(50 \leq N_{\text {run }} \leq 100\right)$ independent simulation runs for each network and for given set of parameters $(q, N)$. Here, were used 50 independent networks for each system size $N$ cited earlier.

Table 1. The critical noise $q_{c}$, the critical exponents, and the effective dimensionality $D_{\text {eff }}$, for DBA, UBA, DER, UER, and SHP network with connectivity $k=4$. Error bars are statistical only. $\gamma / \nu^{q_{c}}$ is calculated from $\chi$ at $q_{c}$ and $\gamma / \nu^{q_{c}(N)}$ from the maximal $\chi$.

\begin{tabular}{|cccccccr|}
\hline \hline$k=4$ & $q_{c}$ & $\beta / \nu$ & $\gamma / \nu^{q_{c}}$ & $\gamma / \nu^{q_{c}(N)}$ & $1 / \nu$ & $D_{\text {eff }}$ & Ref. \\
\hline$D B A$ & $0.431(3)$ & $0.447(2)$ & $0.856(15)$ & $0.888(9)$ & - & $0.998(3)$ & {$[20$} \\
$U B A$ & $0.306(3)$ & $0.231(22)$ & $0.537(8)$ & $0.519(17)$ & $0.43(2)$ & $0.999(23)$ & 21 \\
$D E R$ & $0.175(4)$ & $0.230(5)$ & $0.530(6)$ & $0.516(2)$ & $0.545(26)$ & $0.990(7)$ & 22 \\
$U E R$ & $0.181(1)$ & $0.242(6)$ & $0.54(1)$ & $0.515(6)$ & $0.59(7)$ & $1.02(2)$ & 18 \\
$S H P$ & $0.166(3)$ & $0.230(3)$ & $0.535(2)$ & $0.523(5)$ & $0.475(8)$ & $0.995(3)$ & here \\
\hline \hline
\end{tabular}




\section{Results and Discussion}

In Figs. 1, 2, and 3] we show the dependence of the magnetization $M$, susceptibility $\chi$, and Binder cumulant $U$ on the noise parameter $q$, obtained from simulations on $S H P$ networks with $L$ ranging from $N=250$ to 16000 sites. The shape of $M(q)$, $\chi(q)$, and $U(q)$ curve, for a given value of $N$, suggests the presence of a second-order phase transition in the system. The phase transition occurs at the critical value $q_{c}$ of the noise parameter $q$. This parameter $q_{c}$ is estimated as the point where the $U_{N}(q)$ curves for different system sizes $N$ intercept each other 29 . Then, we obtain $q_{c}=0.166(3)$ and $U^{*}=0.288(3)$ for $S H P$ networks.

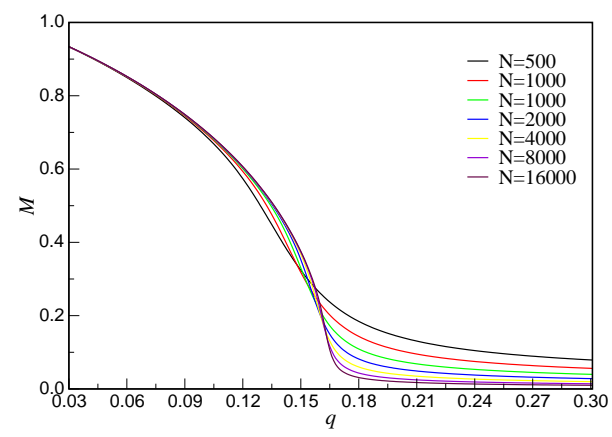

Fig. 1. Plot of the magnetization $M$ as a function of the noise parameter $q$, for $N=250,500$, 1000, 2000, 4000, 8000, and 16000 sites.

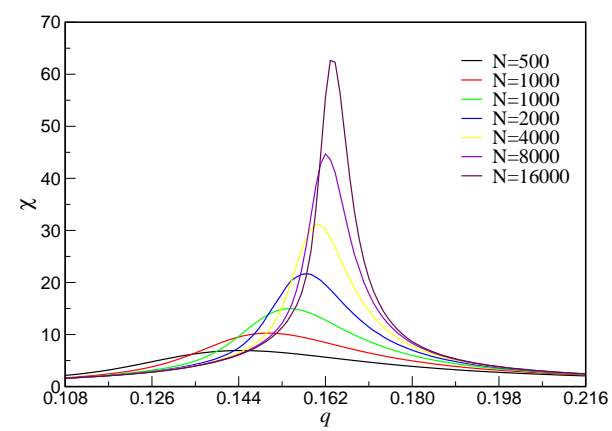

Fig. 2. The same Fig. 1 but now for the susceptibility $\chi$ as a function of the noise parameter $q$.

In Fig. 4 we plot the dependence of the magnetization $M^{*}=M\left(q_{c}\right)$ vs. the system size $N$. The slope of curve corresponds to the exponent ratio $\beta / \nu$ according to Eq. (3a). The obtained exponent is $\beta / \nu=0.230$ (3) for our $S H P$ network. 
The exponent ratio $\gamma / \nu$ at $q_{c}$ is obtained from the slope of the straight line with $\gamma / \nu=0.535(2)$, as presented in Fig. 5. The exponents ratio $\gamma / \nu$ at $q_{\chi_{\max }}(N)$ is $\gamma / \nu=0.523(5)$ for $S H P$ networks as presented in Fig. [6]

To obtain the critical exponent $1 / \nu$, we used the scaling relation (4). The calculated value of the exponent $1 / \nu$ are $1 / \nu=0.475(8)$ for SHP networks (see Fig. 77). We plot $M N^{\beta / \nu}$ versus $\left(q-q_{c}\right) N^{1 / \nu}$ in Fig. 8 using the critical exponents $1 / \nu=0.475(8)$ and $\beta / \nu=0.230(3)$ for system size $N=1000,2000,4000,8000$, and 16000 for $S H P$ network. The excellent collapse of the curves for five different system sizes corroborates the estimate for $q_{c}$ and the critical exponents $\beta / \nu$ and $1 / \nu$.

In Fig. 9 we plot $\chi N^{-\gamma / \nu}$ versus $\left(q-q_{c}\right) N^{1 / \nu}$ using the critical exponents $\gamma / \nu=$ $0.523(5)$ and $1 / \nu=0.475(8)$ for system size $N=1000,2000,4000,8000$, and 16000 for $S H P$ network. Again, the excellent collapse of the curves for five different system size corroborates the extimation for $q_{c}$ and the critical exponents $\gamma / \nu$ and $1 / \nu$. The results of simulations are collected in Tab. 1.

\section{Conclusion}

The determination of the universality class of the MVM model on differents nonregular structure as Small-Worlds, scale-free networks, random graphs, and others has been studied by many researchers in recent years $16|17| 18|19| 20|21| 22 \mid 23$. Finally, here, we remark that our MC results obtained on SHP network for MVM model show that critical exponent ratios $\beta / \nu$ and $\gamma / \nu$ are similar to those for $U B A$ networks, $U E R$ and $D E R$ random graphs for values of conectivity $k=4$, but different from the results of $D B A$ networks and MVM model for regular lattice $\underline{6}$ and equilibrium $2 \mathrm{D}$ Ising model 2 . Here, we show also that the critical exponent $1 / \nu$ is different from random Erdös-Rényi graphs. Therefore, unfortunately, because of the critical exponent ratio $1 / \nu$, we cannot assert that MVM models on different structures as scale-free $(U B A)$ network and random graphs ( $U E R$ and $D E R$ ) belong to the same universality class of the MVM model on $S H P$ network; only the critical exponent ratios $\beta / \nu$ and $\gamma / \nu$ are similar to $U B A$ networks and to $U E R$ and $D E R$ random graphs. Here, we also showed that the effective dimension $D_{\text {eff }}$ is close to 1 for all networks and graphs studied in this work. The agreement in $D_{\text {eff }}$ and the two exponent ratios but not in $1 / \nu$ (weak universality) remains to be explained.

\section{Acknowledgments}

The author thanks D. Stauffer for many suggestion and fruitful discussions during the development this work and also for reading this paper. We also acknowledge the Brazilian agency CNPQ for its financial support. This work also was supported the system SGI Altix 1350 in the computational park CENAPAD.UNICAMP-USP, SP-BRAZIL. 


\section{References}

1. L. Onsager, Phys. Rev. 65, 117 (1944); B. Kaufmann, Phys. Rev. 76, 1232 (1949).

2. R. J. Baxter, Exactly solved models in statistical mechanics, London, Academic Press (1982).

3. B. Latané, Am. Psychologist 36, 343 (1981).

4. C. Castellano, S. Fortunato and V. Loreto, Rev. Mod. Physics 81, 591 (2009) = http://rmp.aps.org/abstract/RMP/v81/i2/p591-1

5. D. Stauffer, J. Stat. Phys. 151, 9 (2013).

6. M. J. Oliveira, J. Stat. Phys. 66273 (1992).

7. G. Zaklan, F. Westerhoff and D. Stauffer, arXiv:0801.2980 (2008), = J. Econ. Interact. Coordination 4, 1 (2008).

8. G. Zaklan, F. W. S. Lima and F. Westerhoff, Physica A 387, 5857 (2008).

9. F. W. S. Lima, Journal of Physics: Conference Series 246, 012033 (2010).

10. F. W .S. Lima, Theoretical Economics Letters 02, 87 (2012).

11. F. W. S. Lima, International Journal of Modern Physics C 23, 1250079 (2012).

12. M. A. Santos, S. Teixeira, J. Stat. Phys. 78, 963 (1995).

13. F. W. S. Lima and K. Malarz, Int. J. Mod. Phys. C 17, 1273 (2006).

14. J. C Santos, F. W. S. Lima, and K. Malarz, Physica A, 390, 359 (2011).

15. F. W. S. Lima, Physica. A, 391, 1753 (2012).

16. P. R. Campos, V. M. Oliveira, and F. G. B. Moreira, Phys. Rev. E 67, 026104 (2003).

17. E. M. S. Luz, F. W. S. Lima, Int. J. Mod. Phys. C 18, 1251 (2007).

18. L. F. C. Pereira and F. G. B. Moreira, Phys. Rev. E 71, 016123 (2005).

19. F. W. S. Lima, U. L. Fulco, and R. N. C. Filho, Phys. Rev. E 71, 036105 (2005).

20. F. W. S. Lima, Int. J. Mod. Phys. C 17, 1257 (2006).

21. F. W. S. Lima, Communications in Computational Physics 2, 358 (2007).

22. F. W. S. Lima, A. O. Sousa, and M. A. Sumour, Physica A 387, 3503 (2008)

23. F. W. S. Lima, A. A. Moreira, A. D. Araújo, Phys. Rev. E 86, 056109 (2012).

24. N. A. M. Araújo, J. S. Andrade Jr., H. J. Herrmann, "Tactical Voting in Plurality Elections", PLoS ONE 5(9) (2010): e12446. doi:10.1371/journal.pone.0012446.

25. F. W. S. Lima, T. Hadzibeganovic and D. Stauffer, Physica A 388, 4999 (2009).

26. T. Qiu, T. Hadzibeganovic, G. Chen, L.-X. Zhong and X.-R. Wu, Computer Phys. Comm. 181, 2057 (2010).

27. D. Stauffer, M. Hohnisch and S. Pittnauer, Physica A 370, 734 (2006). See also P. Holme and M. E. J. Newman, Phys. Rev. 74, 056108 (2006); A. E. Allahveryan and K. G. Petrosyan, Europhysics Letters 75, 908 (2006).

28. In preparation, "Majority-vote model with 2 and 3 states on SHP networks".

29. K. Binder and D. W. Heermann, Monte Carlo Simulation in Statistical Phyics, (Springer Verlag, 1988). 


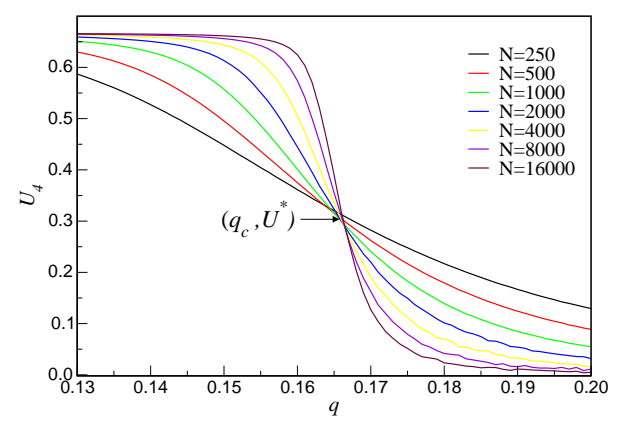

Fig. 3. The same the Fig. 1 but now for Binder cumulant $U$ as a function of the noise parameter $q$.

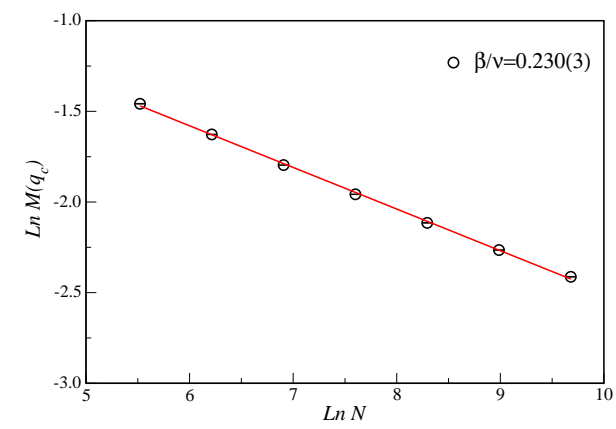

Fig. 4. Plot of the magnetization $M^{*}=M\left(q_{c}\right)$ vs. the linear system size $N$ SHP network.

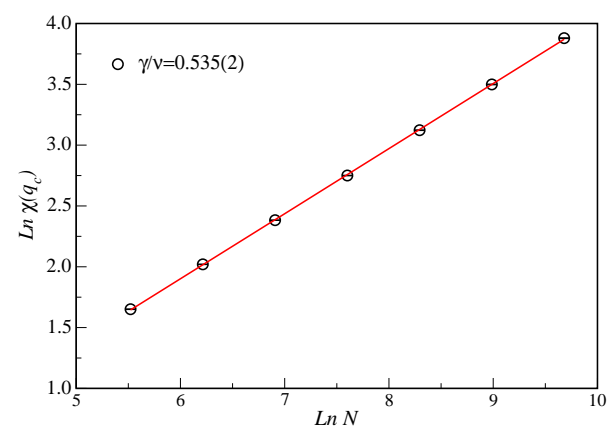

Fig. 5. Display of the susceptibility at $q_{c}$ versus $N$ for SHP network. 


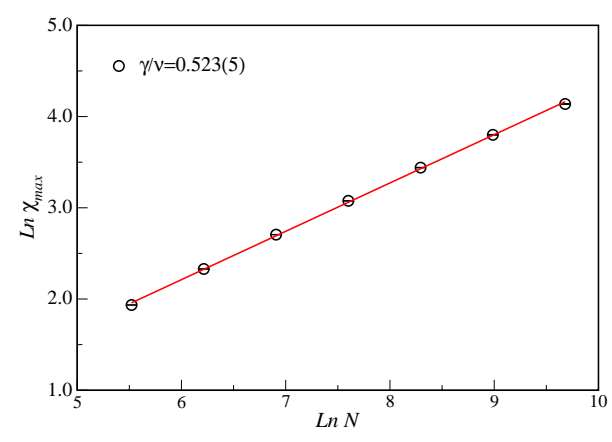

Fig. 6. Plot of the susceptibility at $q_{\chi_{\max }}(N)$ versus $N$ for for SHP network.

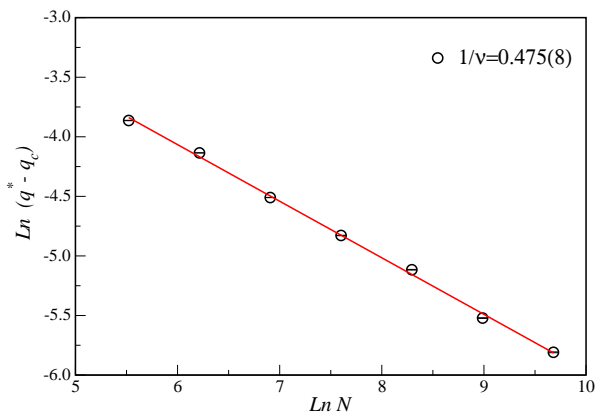

Fig. 7. Plot $\ln \left|q_{c}(N)-q_{c}\right|$ versus the system size $N$ for SHP network.

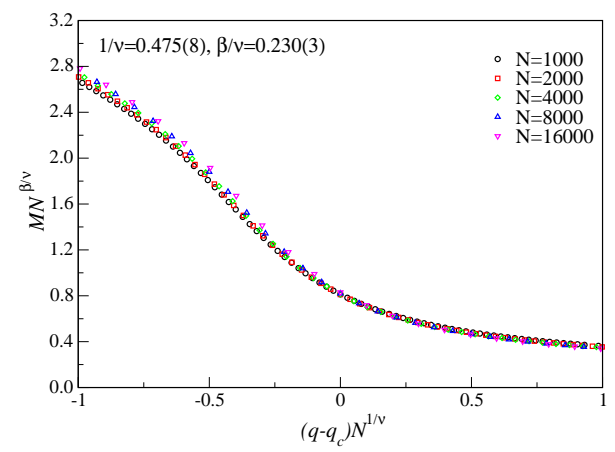

Fig. 8. Plot of the data collapse of the magnetisation $M$ for the system size $N=1000,2000$, 4000,8000 , and 16000 for $S H P$ network. The exponents used here were $\beta / \nu=0.230(3)$ and $1 / \nu=0.475(8)$. 


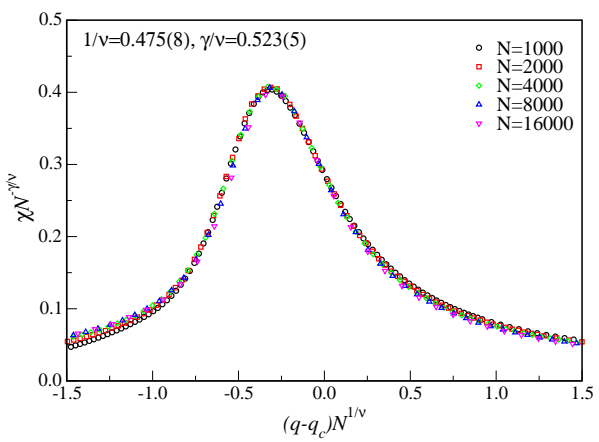

Fig. 9. Data colapse of the susceptibility for the system size $N=1000,2000,4000,8000$, and 16000 for $S H P$ network. The exponents used here were $\gamma / \nu=0.523(5)$ and $1 / \nu=0.475(8)$. 